\title{
Changes of Gait Characteristics in Stroke Patient with Foot Drop After Treating by Foot Drop Stimulator Combined with Moving Treadmill Training
}

Peishun CHEN ( $\nabla$ cpshun21@126.com )

Guangzhou Panyu Central Hospital https://orcid.org/0000-0002-3504-2097

Haiwang ZHOU

Guangzhou Panyu Central Hospital

Taotao LI

Guangzhou Panyu Central Hospital

Hongli GUANG

Guangzhou Panyu Central Hospital

Yu MIN

Guangzhou Panyu Central Hospital

Wanrong ZHANG

Guangzhou Panyu Central Hospital

\section{Research}

Keywords: foot drop stimulator, moving treadmill, stroke, foot drop, gait

Posted Date: August 3rd, 2021

DOl: https://doi.org/10.21203/rs.3.rs-757627/v1

License: (c) (1) This work is licensed under a Creative Commons Attribution 4.0 International License.

Read Full License 


\section{Abstract}

\section{Background and Purpose}

To analyze the changes of gait characteristics in stroke patient with foot drop after treating by foot drop stimulator combined with moving treadmill, and provide a basis for the improvement of foot drop gait after stroke.

\section{Methods}

Sixty patients with stroke and hemiplegia with foot drop were randomly divided into the experimental group and the control group. Both groups received basic rehabilitation training. On this basis, the experimental group received foot drop stimulator combined with moving treadmil. The control group received foot drop stimulator training. The treatment time of the two groups was 30 minutes/time, 5 times a week, for 3 consecutive weeks. Gait Watch three-dimensional gait analysis system was used to record the angle of hip flexion, knee flexion and ankle flexion before and after treatment.

\section{Results}

After treatment, the angles of hip flexion, knee flexion, and ankle flexion of the two groups of patients changed, but the above changes in the experimental group were better than those in the control group, The difference is statistically significant $(p<0.05)$.

\section{Conclusions}

The foot drop stimulator combined with moving treadmill can significantly improve the foot gait after stroke, and promote the normalization of hip flexion and knee flexion.

\section{Introduction}

Foot drooping gait after stroke is very common, with an incidence rate as high as $20 \%-30 \%, 1,2$ which manifests as ankle joints are not flexed or insufficiently flexed, showing a cross-threshold gait when walking, affecting walking efficiency. ${ }^{3,4}$ This study found that the foot drop stimulator combined with moving treadmill can effectively improve the foot-dropping gait, improve ankle flexion, hip flexion and knee flexion after stroke.

\section{Methods}

60 patients with stroke and hemiplegia who were treated in our hospital from January 2019 to March 2020 were selected, divide into the experimental group and the control group with 30 cases each, and the inclusion criteria were: $\triangle$ All patients met the diagnostic criteria for stroke in the "2016 Guidelines and Consensus on Diagnosis and Treatment of Chinese Cerebrovascular Diseases" ${ }^{5}$ and confirmed by

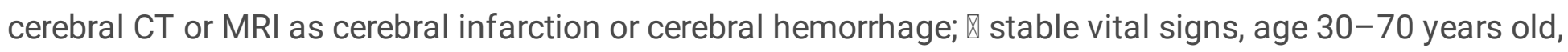


course 15 days-3 months; 区hemiplegia in one limb, walking ability classification (Funtional ambulation category, FAC ) $\geq 1$ grade; खpresence of foot drop gait, improved muscular tone of the triceps on the hemiplegic side of the lower leg triceps (flatus, gastrocnemius) $\leq 2$ grades, no serious cognitive dysfunction. Exclusion criteria: 『Cerebellar stroke; 『Severe cognitive impairment; $邓$ Four limbs paralysis; $\triangle$ Parkinson's disease; 囚Lower limb fracture; $₫$ Recent venous thrombosis of the lower limb; $₫$ Recent

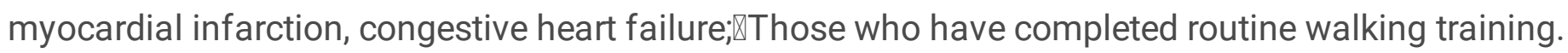
There were no statistical differences in gender, age, disease course, and stroke stratification between the two groups (Table 1).

Table 1

Comparison of 2 groups of general information

\begin{tabular}{|c|c|c|c|c|c|c|c|}
\hline \multirow[t]{2}{*}{ Team } & \multirow[t]{2}{*}{$\mathbf{n}$} & \multicolumn{2}{|c|}{$\operatorname{Sex}(n)$} & \multirow{2}{*}{$\begin{array}{l}\text { Age(year } \\
\text { old) }\end{array}$} & \multirow{2}{*}{$\begin{array}{l}\text { Course of } \\
\text { disease(Days) }\end{array}$} & \multicolumn{2}{|c|}{ Stroke stratification(n) } \\
\hline & & Boy & Girl & & & $\begin{array}{l}\text { Cerebral } \\
\text { infarction }\end{array}$ & $\begin{array}{l}\text { Cerebral } \\
\text { hemorrhage }\end{array}$ \\
\hline $\begin{array}{l}\text { Test } \\
\text { group }\end{array}$ & 30 & 17 & 13 & $\begin{array}{l}58.57 \pm \\
8.47\end{array}$ & $44.37 \pm 19.99$ & 20 & 10 \\
\hline $\begin{array}{l}\text { Control } \\
\text { group }\end{array}$ & 30 & 16 & 14 & $\begin{array}{l}58.20 \pm \\
7.98\end{array}$ & $44.23 \pm 19.50$ & 19 & 11 \\
\hline$x^{2} / t$ & & 0.06 & & 0.172 & 0.027 & 0.073 & \\
\hline P & & 0.79 & & 0.864 & 0.979 & 0.787 & \\
\hline
\end{tabular}

All patients received routine rehabilitation training, that is, neuromuscular promotion techniques were given to the patients according to their functional status. On this basis, for foot drop, the experimental group received foot drop stimulator combined with moving treadmill, that is, walking training was carried out synchronously on the activity plate using the programmed electrical stimulation of the foot drop stimulator; the control group received foot drop stimulator training, the treatment time of the two groups was 30 minutes/time, 5 times a week, for 3 weeks. This study was approved by the Research Ethics Committee of Guangzhou Panyu Central Hospital. All individuals provided written informed consent before participation in this study.

\section{Foot drop stimulator}

XFT-2001 Foot drop stimulator(XFT) produced by Shenzhen Xunfengtong Electronics Co., Ltd. Before treatment, use neuromuscular locator to locate the sensitive position on the anterior tibial muscles. Connect the main unit and use the walking mode. When the affected leg moves forward, the electrical stimulation is started to stimulate the anterior tibial muscle to produce dorsiflexion; when the heel is on the ground, the electrical stimulation is turned off. So that the patient can flexion of the foot in time during walking, and the patient's walking is more stable, more natural and safer.

\section{Moving treadmill training (MTT)}


Moving treadmill training (MTT) is a task-oriented and supportive training. In active treadmill training, the patient walks at a set speed with the help of a flat track, just like walking in place, without being affected by space, can exhibit a more symmetrical walking posture.

Gait Watch three-dimensional gait analysis system was used to record the angle of hip flexion, knee flexion and ankle flexion before and after treatment.

\section{Outcomes and statistical analyses}

The data obtained before and after the treatment were represented by " $\pm S$ ", and the SPSS 22.0 statistical software package was used for data analysis. The comparison count data within the group and between groups were tested by $\chi 2$, the measurement data were tested by $T$, and the test level was $a=0.05, p<0.05$ represented The difference was statistically significant.

\section{Results}

After 3 weeks of treatment, the angles of hip flexion, knee flexion, and ankle flexion of the two groups of patients all changed, but the above changes in the experimental group were better than those in the control group, and the difference was statistically significant $(p<0.05$, Table 2$)$

Table 2

Change of angle of hip flexion, knee flexion and ankle flexion

\begin{tabular}{|llllllll|}
\hline \multirow{2}{*}{ Team } & $\mathbf{n}$ & \multicolumn{2}{l}{ Hip flexion $\left({ }^{\circ}\right)$} & \multicolumn{2}{l|}{ Knee flexion $\left({ }^{\circ}\right)$} & \multicolumn{2}{l|}{ Ankle flexion $\left({ }^{\circ}\right)$} \\
\cline { 3 - 8 } & & Before & After & Before & After & Before & After \\
\hline Test group & 30 & $\begin{array}{l}10.93 \pm \\
4.18\end{array}$ & $20.80 \pm$ & $30.97 \pm$ & $44.87 \pm$ & $4.57 \pm$ & $12.17 \pm$ \\
& & 4.62 & 10.69 & 13.16 & 3.61 & 3.43 \\
\hline $\begin{array}{l}\text { Control } \\
\text { group }\end{array}$ & 30 & $\begin{array}{l}10.73 \pm \\
4.03\end{array}$ & $16.97 \pm$ & $30.90 \pm$ & $38.10 \pm$ & $4.23 \pm$ & $5.57 \pm$ \\
$\mathrm{t}$ & & 0.189 & 4.293 & 0.024 & 2.156 & 0.349 & 6.808 \\
$\mathrm{p}$ & 0.851 & 0.000 & 0.981 & 0.035 & 0.729 & 0.000 \\
\hline
\end{tabular}

\section{Discussion}

After stroke, the patient is prone to induce abnormal postures such as "circling gait" because of foot drop. Walking in this abnormal exercise mode will hinder the establishment of normal exercise mode and affect the recovery of lower limb motor function in hemiplegia. ${ }^{6}$ The ankle joint is the adjustment center of human walking posture and stability. Its dorsiflexion function plays an important role in gait. Abnormal dorsiflexion function will affect the angle of hip flexion and knee flexion, resulting in abnormal gait such as sagging. ${ }^{7}$ 
Foot drop after stroke is mainly caused by central nervous system injury. ${ }^{8}$ Under normal circumstances, the dorsiflexion of the ankle joint is controlled by the common peroneal nerve. There are two branches of the common peroneal nerve, which control the anterior calf muscle group (tibialis anterior muscle) and lateral calf muscle group (long fibula and short muscle). The XFT-2001 foot drop stimulator stimulates the tibialis anterior and fibula long and short muscles by designing a reasonable and targeted electrical stimulation, causing them to contract, and makes the ankle joint dorsiflexed and valgus, thus correcting the foot drop and foot inversion. ${ }^{9}$ The correction of foot inversion and foot drop stimulator is helpful for the clearance of the swinging ankle joint during the gait cycle and reducing the body's compensatory posture. ${ }^{10}$ The author's previous research results also confirmed that the foot drop walker can effectively improve walking function and lower extremity motor function, reduce the difference between left and right step length, and increase the pace. ${ }^{11}$ However, the use of a foot drop walker requires patients to have a certain standing balance and walking ability, and requires a sufficiently wide walking training field, which limits the use of the device.

Moving treadmill training is a task-oriented and supportive training. In the activity flat training, the patient walks at the set speed by the flat track, just like walking in place, not limited by the venue and space, can be expressed a more symmetrical walking posture to improve walking speed and endurance. ${ }^{12}$ Moving treadmill training can solve the patient's lack of balance function and the limitation of the training venue. In recent years, it has been more used for walking function training. ${ }^{13,14}$ However, if the patient has foot drop, when simply using moving treadmill to walk, due to the constant speed of the crawler, it will be prone to sprained ankle.

In this study, the foot drop stimulator combined with Moving treadmill training made up for the shortcomings of the two methods above, and fully showed their respective advantages. The results of the study showed that after treatment, the experimental group's angles of hip flexion, knee flexion, and ankle flexion in the affected side changed significantly during the gait cycle. After treatment, the ankle dorsiflexion angle increased $(p<0.001)$, indicating that the motor function of the tibialis anterior muscle was restored and the muscle strength was enhanced, which was benefited from the stimulation effect of the foot drop walker on the tibialis anterior muscle. The improvement of ankle dorsiflexion function and the movement of the active flat track can help promote the flexion of the knee joint, so the knee flexion angle increases after treatment $(p<0.001)$. The improvement of the ankle dorsiflexion function and the driving of the movable flat track help to promote hip flexion and knee flexion, so the angle of hip flexion and knee flexion increased after treatment $(p<0.05)$.

To sum up, the combination of foot drop stimulator and moving treadmill can promote the recovery of lower limb motor function, improve foot sag, ankle flexion, knee flexion and hip flexion ability, thereby improving foot drop gait.

\section{Abbreviations}

Funtional ambulation category, FAC 
XFT-2001 Foot drop stimulator, XFT

Moving treadmill training, MTT

\section{Declarations}

\section{Ethics approval and consent to participate}

This study was approved by the Research Ethics Committee of Guangzhou Panyu Central Hospital. खPan Yi Lun Approval [2018] No. 2区

\section{Consent for publication}

Agree

\section{Availability of data and material}

Please contact author for data requests. Corresponding Author: Chen Peishun, e-mail: cpshun21@126.com

\section{Competing interests}

The authors declare that they have no competing interests.

\section{Funding}

This research was supported by a fund (2018-Z04-06) by Key Discipline Construction Fund of Guangzhou Panyu Science and Information Bureau.

\section{Authors' contributions}

Chen Peishun carried out the studies, drafted the manuscript. Zhang Wanrong participated in case collection. Li Taotao participated in assessment and patient treatment. Guan Hongli participated in the design of the study and performed the statistical analysis. Min Yu conceived of the study, and participated in its design and coordination and helped to draft the manuscript. Zhou Haiwang helped to draft the manuscript. All authors read and approved the final manuscript.

\section{Acknowledgements}

The authors' institution is department of rehabilitation medicine, guangzhou panyu central hospital.

\section{References}

1. Rakesh P , Arvind R, Nolan K J . Alterations in Spectral Attributes of Surface Electromyograms after Utilization of a Foot Drop Stimulator during Post-Stroke Gait[J]. Frontiers in Neurology, 2017, 8:449-. 
2. Aurore, Thibaut, Florent, Moissenet, Carol, \& Di, et al. Brain plasticity after implanted peroneal nerve electrical stimulation to improve gait in chronic stroke patients: two case reports. NeuroRehabilitation, 2017,40(2):25I-258.

3. Liang Haitang, Zeng Xuan, Huang Yugui, Wu Qiaoyun. [Effects of acupuncture combined with exercise rehabilitation training on foot ptosis in the recovery stage of stroke]. Chin Jo Geront. 2017,37(12):2916-2918. Chinese.

4. Wang Yanxue, Cao Haijie, Sun Lepeng, Li Shunming, Wang Yanan, Yang Qi, et,al. [Observation of the therapeutic effect of repeated transcranial magnetic stimulation combined with myoelectric biofeedback electrical stimulation therapy on foot prolapse after stroke]. Chin Rehabil. 2019,34(3): 119-122. Chinese.

5. Editor-in-chief of the Neurology Branch of the Chinese Medical Association. 2016 edition of guidelines and consensus for diagnosis and treatment of Chinese cerebrovascular diseases. Beijing: People's Medi Pub, 2016,1-5.Chinese.

6. Li Weili,Tong Lin,Zhang Wenjie. [Efficacy of action observation therapy based on mirror neuron theory in patients with foot drop after stroke]. Chin J of Rehabil. 2019, 34(5):569-572.Chinese.

7. Sheng Yilan, Ran Jun, Hu Guojiong, Lu Shouxing, Zhao Yunchao, Qu Qi. [Immediate curative effect observation of intramuscular patch to improve walking function of patients with foot prolapse after stroke]. Chin J Sports Med. 2019,38(9):802 -805.Chinese.

8. Shaikh, Faraz, M. , Salcic, Zoran, Wang, \& I-Kai, K. , et al. Bipedal gait model for precise gait recognition and optimal triggering in foot drop stimulator: a proof of concept[J]. Med Biol Eng Comput, 2018,56(9):1731-1746ه

9. Chen Peishun, Li Hao, Li Taotao, Huang Zhen, Zhou Haiwang. [Clinical observation of intramuscular patch combined with foot drop aid to treat foot drop gait in patients with stroke hemiplegia].Ner In Fun Rec.2016,11(06) :545-546.Chinese.

10. Yang Ting, Li Xueping, Lin Qiang. [The effect of gait-induced functional electrical stimulation on walking ability of patients with hemiplegic foot ptosis]. Chin J of Rehabil.2018,33(2):170174. Chinese.

11. Chen Peishun, Huang Zhen, Li Hao, Min Yu, Qu Fei, Zhou Haiwang. [The effect of virtual reality combined with active plate training on walking function of stroke patients]. Ner In Fun Rec. 2015, (4):360-361.Chinese.

12. Shi Meifang, Wu Hua, Gu Xudong, Fu Jiangming, Yao Yunhai, Wu Caihong, et,al.[ The effect of exercise feedback combined with weight loss plate training on gait recovery in stroke hemiplegic patients]. Chin J of Rehabil. 2016,38 (8):612-614.Chinese.

13. Shirin, Tajali, Mohammad, Mehravar, Hossein, Negahban, etc, al. Impaired local dynamic stability during treadmill walking predicts future falls in patients with multiple sclerosis: A prospective cohort study. Clinical biomechanics (Bristol, Avon),2019,67:197-201.

14. Y Liu, X Zang, N Zhang, M Wu. Gait Symmetry Can Reduce Dependence on the Intact Limb during Walking with Constraint of Unilateral Metatarsophalangeal Joints.Conference proceedings: Annual 
International Conference of the IEEE Engineering in Medicine and Biology Society. IEEE Engineering in Medicine and Biology Society. Annual Conference,2018:2300-2303.

\section{Supplementary Files}

This is a list of supplementary files associated with this preprint. Click to download.

- Data.xlsx 